\title{
Discovery and full genome characterization of a new SIV lineage infecting red-tailed guenons (Cercopithecus ascanius schmidti) in Kibale National Park, Uganda
}

Michael Lauck ${ }^{1}$, William M Switzer ${ }^{2}$, Samuel D Sibley ${ }^{3}$, David Hyeroba ${ }^{4}$, Alex Tumukunde ${ }^{4}$, Geoffrey Weny ${ }^{4}$, Anupama Shankar ${ }^{2}$, Justin M Greene ${ }^{1}$, Adam J Ericsen ${ }^{1}$, HaoQiang Zheng ${ }^{2}$, Nelson Ting ${ }^{5}$, Colin A Chapman ${ }^{4,6,7}$, Thomas C Friedrich ${ }^{1,3}$, Tony L Goldberg ${ }^{1,3,4}$ and David H O'Connor ${ }^{1,8^{*}}$

\begin{abstract}
Background: Human immunodeficiency virus (HIV) type 1 and 2, the causative agents of acquired immunodeficiency syndrome (AIDS), emerged from African non-human primates (NHPs) through zoonotic transmission of simian immunodeficiency viruses (SIV). Among African NHPs, the Cercopithecus genus contains the largest number of species known to harbor SIV. However, our understanding of the diversity and evolution of SIVs infecting this genus is limited by incomplete taxonomic and geographic sampling, particularly in East Africa. In this study, we screened blood specimens from red-tailed guenons (Cercopithecus ascanius schmidti) from Kibale National Park, Uganda, for the presence of novel SIVs using unbiased deep-sequencing.

Findings: We describe and characterize the first full-length SIV genomes from wild red-tailed guenons in Kibale National Park, Uganda. This new virus, tentatively named SIVrtg_Kib, was detected in five out of twelve animals and is highly divergent from other Cercopithecus SIVs as well as from previously identified SIVs infecting red-tailed guenons, thus forming a new SIV lineage.

Conclusions: Our results show that the genetic diversity of SIVs infecting red-tailed guenons is greater than previously appreciated. This diversity could be the result of cross-species transmission between different guenon species or limited gene flow due to geographic separation among guenon populations.
\end{abstract}

Keywords: Simian immunodeficiency virus, SIV, Non-human primates, Guenons, Uganda, Kibale National Park

\section{Findings}

Simian immunodeficiency viruses (SIV) naturally infect at least 45 different African non-human primate (NHP) species [1]. Zoonotic transmission of SIVs has led to the emergence of human immunodeficiency virus type 1 (HIV-1) and type 2 (HIV-2) [2,3]. Only African Old World monkeys (OWM) and apes from sub-Saharan Africa, but not their Asian counterparts or New World monkeys, are naturally infected with SIV. The Cercopithecus genus, also

\footnotetext{
* Correspondence: david.h.oconnor@gmail.com

'Wisconsin National Primate Research Center, 555 Science Dr, 53705

Madison, WI, USA

${ }^{8}$ Department of Pathology and Laboratory Medicine, University of

Wisconsin-Madison, Madison, WI, USA

Full list of author information is available at the end of the article
}

termed guenons, is of special interest among OWM since it comprises the largest number of species known to harbor SIV [4]. Surprisingly, SIVtal, isolated from a talapoin monkey (Miopithecus ogouensis), a different primate genus, also sorts with Cercopithecus SIVs, suggesting either evolution from a common ancestor or transmission between genera [5]. Although guenons occupy all of subSaharan Africa [6], the vast majority of full-length SIV sequences have been obtained from West and Central African monkeys, potentially influencing our current understanding of the diversity and evolutionary history of Cercopithecus SIVs.

Here, we report the discovery and characterization of a novel SIV lineage infecting red-tailed guenons 
(Cercopithecus ascanius schmidti) from Kibale National Park (KNP), the same location where we previously reported SIV in red colobus and black-and-white colobus $[7,8]$. In 2010, we sampled 12 Kibale red-tailed guenons as part of a larger study of primate ecology, conservation, and health belonging to at least three social groups [9]. All samples were collected within an area of approximately $15 \mathrm{~km}^{2}$. Animals were anesthetized and samples were collected as previously described [10]. Plasma samples were then screened for SIV/HIV antibody reactivity using the INNO-LIA HIV-1/2 Score and HIV-2-based Genelabs western blot assays. Viral RNA was prepared from blood plasma for random hexamer-based sequencing as previously described [11]. By deep sequencing, SIV RNA was detected in three out of twelve red-tailed guenons (Table 1). Antibody reactivity was observed in each SIVpositive animal, with the exception of RT03, possibly indicating an acutely infected animal that had not yet mounted an antibody response or insufficient antibody cross-reactivity to the HIV antigens used in serological tests. In two red-tailed guenons (RT04 and RT05), we detected seroreactivity to at least two viral proteins but were unable to recover SIV-specific reads through deepsequencing (Table 1). We therefore performed RT-PCR, targeting a 400-bp fragment in the C-terminal half of the polymerase $(p o l)$ gene. A faint product was amplified from both plasma samples and the presence of SIVrtg_Kib was subsequently confirmed by Sanger sequencing (KJ865607KJ865608). Overall, infection of SIVrtg_Kib in red-tailed guenons was detected in five out of twelve animals, with no restriction to any one social group. This prevalence is comparable to other non-vpu carrying guenons, although the $95 \%$ confidence interval of our estimate is wide $(19.3 \%$ to $68.1 \%)[4,12-17]$.

De novo assembly of sequence reads yielded complete SIV coding genomes from three individuals (RT03, RT08 and RT11). The sequence of the de novo-assembled genomes was confirmed by deep-sequencing on the Illumina MiSeq, as previously described, resulting in 127,500 176,000 reads (average coverage $1750 \times-2430 \times$ ) mapping to the respective genomes (Figure 1B). The five SIVrtg_Kib genomes were highly similar to each other, sharing $96.9 \pm$ $1.1 \%$ identity at the nucleotide level (Table 2).

A query against the NCBI GenBank database [18] revealed that the new virus was most similar to SIVmus-1, an SIV previously discovered in mustached monkeys (Cercopithecus cephus) from Cameroon [12]. This finding was confirmed by pairwise alignment of the coding regions of SIVrtg_Kib and representatives from major SIV lineages (Table 3). The genomic structure of SIVrtg_Kib is similar to that of other SIVs, including all three structural genes ( $g a g, p o l$ and env) as well as accessory genes (vif, vpr, tat, rev and nef) (Figure 1A). Several Cercopithecus SIVs are characterized by the presence of a $v p u$ gene (SIVmus, SIVmon, SIVgsn, SIVden). However, like SIVdeb, SIVsyk and SIVtal, SIVrtg_Kib does not encode a vpu homolog. Functional motifs in Env and Gag resemble those of other Cercopithecus SIVs, containing 18 cysteine residues in the extracellular glycoprotein gp120 as well as both the PT/ SAP and YPXL budding motifs in the Gag protein [19].

Table 1 Infection of red-tailed (RT) guenons with a novel SIV in Kibale National Park, Uganda,2

\begin{tabular}{|c|c|c|c|c|c|c|c|c|}
\hline \multirow[t]{2}{*}{ Animal } & \multirow[t]{2}{*}{ Sex } & \multicolumn{3}{|c|}{ HIV-2 WB } & \multicolumn{3}{|c|}{ HIV-1/2 InnoLIA } & \multirow[t]{2}{*}{ SIV deep-sequencing ${ }^{3}$, RT-PCR results ${ }^{4}$} \\
\hline & & Gag & Pol & Env & Gag & Pol & Env & \\
\hline RT01 & $\mathrm{F}$ & - & - & - & - & - & - & - \\
\hline RT02 & M & - & - & - & - & - & - & - \\
\hline RT03 & $\mathrm{F}$ & - & - & - & - & - & - & SIVrtg_Kib + (9473) \\
\hline RT04 & $\mathrm{F}$ & $w(p 26)$ & - & w (gp80) & - & - & - &,- RT-PCR + \\
\hline RT05 & $\mathrm{F}$ & $+(p 26)$ & - & $+(\mathrm{gp} 80)$ & $1+(p 24)$ & - & $3+(g p 36)$ & -, RT-PCR + \\
\hline RT06 & M & - & - & - & - & - & - & - \\
\hline RT08 & $\mathrm{F}$ & $+(\mathrm{p} 26)$ & - & w (gp80) & $3+(p 24)$ & - & $3+(g p 120)$ & SIVrtg_Kib + (1932) \\
\hline RT09 & M & $w(p 26)$ & - & - & - & - & - & - \\
\hline RT10 & M & - & - & - & - & - & - & - \\
\hline RT11 & $\mathrm{F}$ & - & - & w (gp80) & - & - & - & SIVrtg_Kib + (5618) \\
\hline RT12 & M & - & - & - & - & - & - & - \\
\hline RT13 & M & - & - & - & - & - & - & - \\
\hline
\end{tabular}

${ }^{1}$ SIV infection was assessed by deep-sequencing of reverse transcribed viral RNA and with serological tests, including HIV-2-specific western blots (HIV-2 WB) and HIV InnoLIA assays.

${ }^{2}$ For HIV-2 WB and HIV-1/-2 InnoLIA, seroreactivity to specific viral proteins is shown in brackets. For the HIV-1/-2 InnoLIA assay, intensity of signals was quantified with " $1+$ " being positive and " $3+$ " being strongly positive. For the HIV-2 WB, intensity of signals was quantified with " $w$ " being weak and " + " being positive.

${ }^{3}$ To quantify the number of SIV-specific reads, read numbers were normalized to 1 million reads per animal.

${ }^{4} \mathrm{~A} 400$-bp fragment of the C-terminal half of the polymerase ( $\mathrm{pol}$ ) gene was amplified by RT-PCR and the presence of SIVrtg_Kib was subsequently confirmed by Sanger sequencing. 


\section{A}

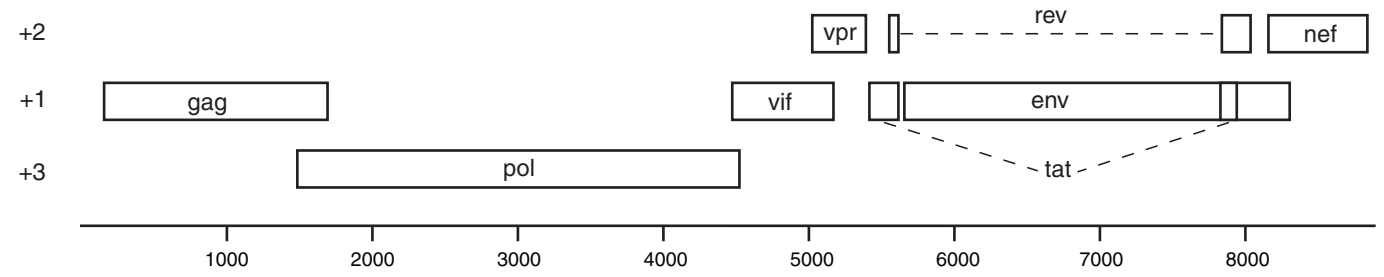

B

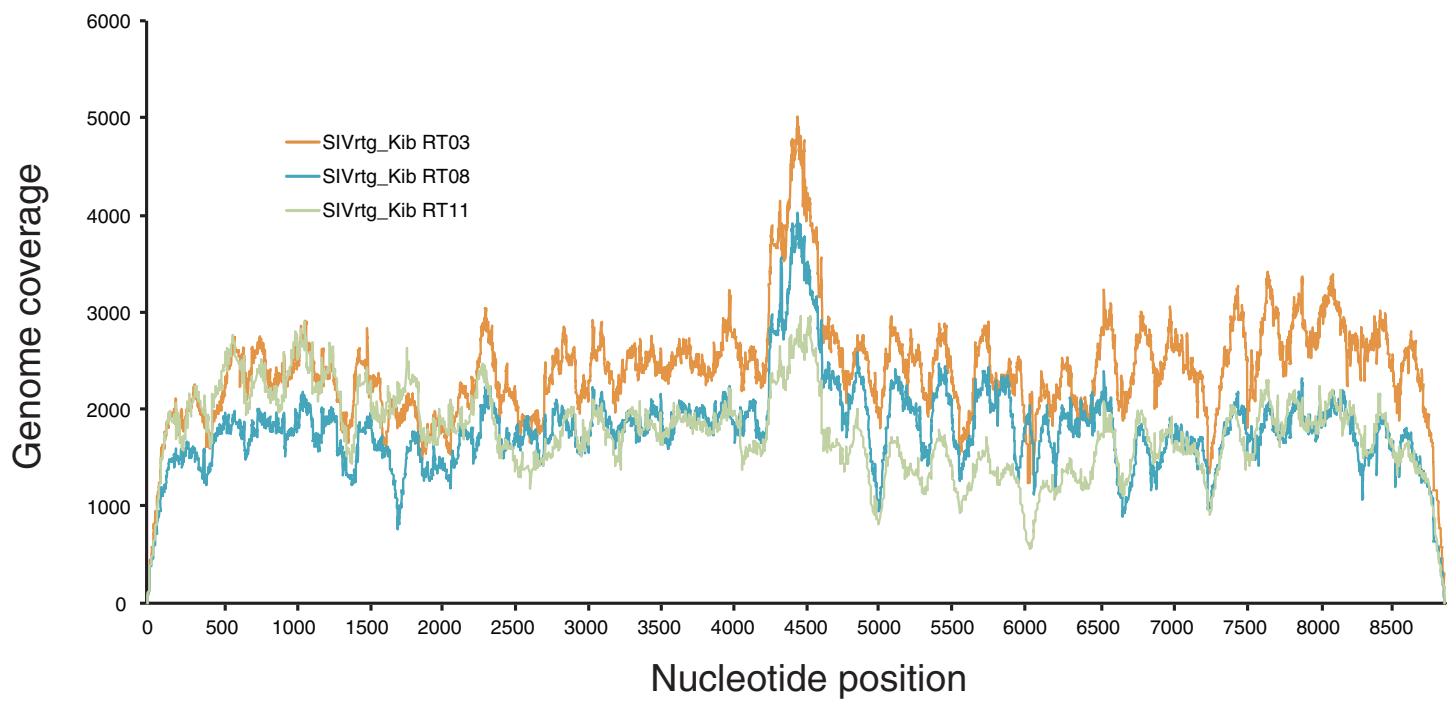

C

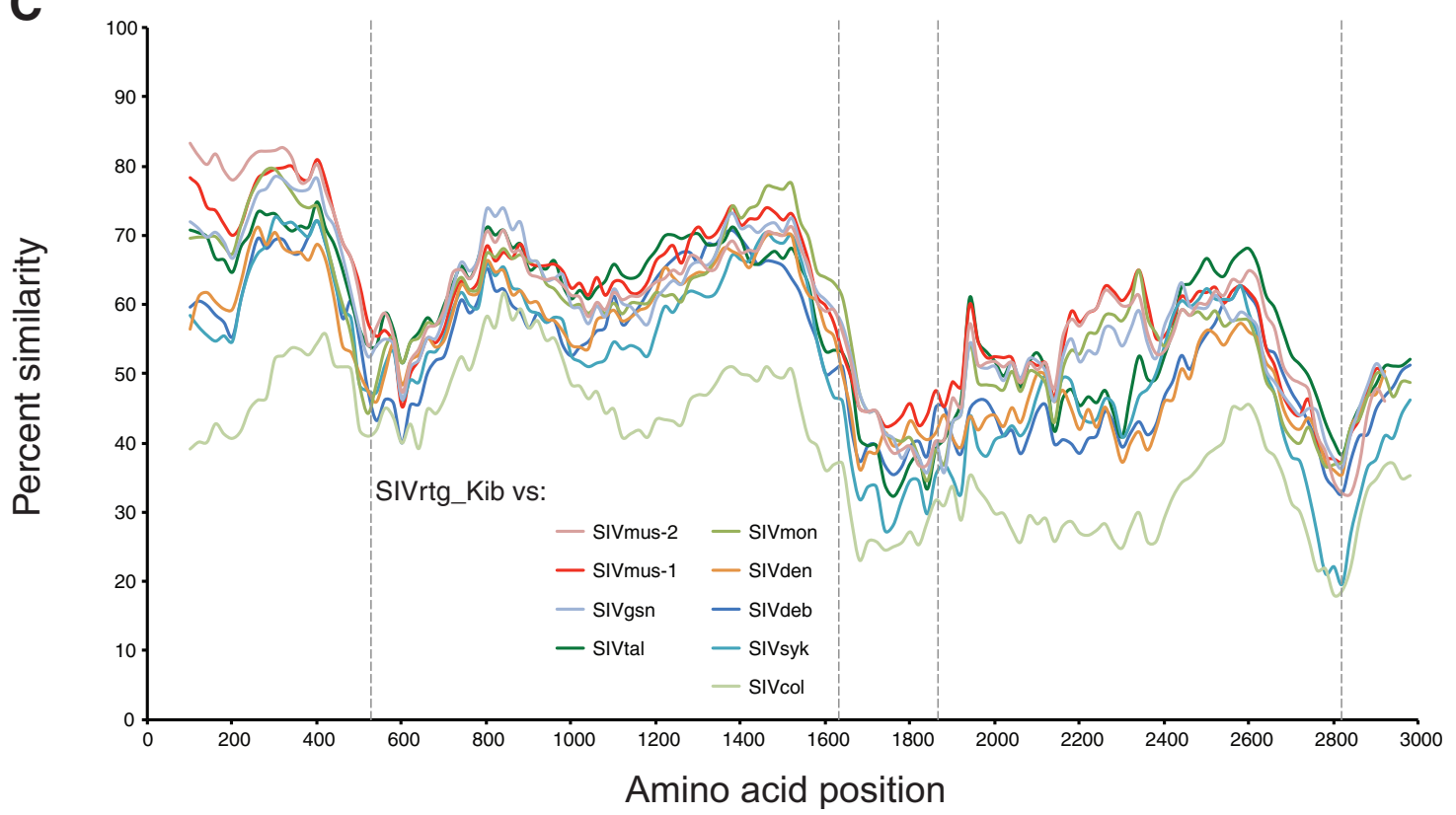

Figure 1 Genomic organization, deep-sequencing genome coverage and similarity plot analysis of SIVrtg_Kib. A: Genome organization of SIVrtg_Kib. Boxes represent open reading frames and are drawn to scale in their respective frame. The rev and tat splice variants are indicated by dashed lines. B: The SIVrtg_Kib genomes from RT03, RT08 and RT11 were deep-sequenced with four overlapping amplicons. The read coverage at each nucleotide position is shown across the genome. C: Sliding window similarity plots of concatenated protein sequences showing the percent similarity of SIVrtg_Kib against other members of Cercopithecus SIVs and SIVcol. Dashed vertical lines indicate start positions of viral proteins Gag, polymerase (Pol), Vif, envelope (Env), and Nef. 
Table 2 Percent nucleotide identity for the five different SIVrtg_Kib isolates ${ }^{1}$

\begin{tabular}{cccccc}
\hline \multicolumn{6}{c}{ Nucleotide identity [\%] } \\
\hline SIVrtg_Kib & $\mathbf{0 3}$ & $\mathbf{0 4}$ & $\mathbf{0 5}$ & $\mathbf{0 8}$ & $\mathbf{1 1}$ \\
$\mathbf{0 3}$ & 100 & & & & \\
$\mathbf{0 4}$ & 99.6 & 100 & & & \\
$\mathbf{0 5}$ & 96.3 & 96.7 & 100 & & \\
$\mathbf{0 8}$ & 95.5 & 95.9 & 95.1 & 100 & \\
$\mathbf{1 1}$ & 97.9 & 98.4 & 97.5 & 96.7 & 100 \\
\hline
\end{tabular}

${ }^{1}$ The percent nucleotide identity is based on a 243 bp polymerase alignment of the 5 isolates.

We analyzed the amino-acid similarity of the novel SIV with related SIV lineages and SIVcol across Gag, Pol, Vif, Env and Nef using SimPlot v3.5.1 [20], following TranslatorX alignment (MAAFT) [21]. Based on the SimPlot analysis, SIVrtg_Kib seems to be equidistantly related to the other members of the Cercopithecus genus across Pol, Vif, Env and Nef, while the Gag protein shares the highest sequence identity with SIVs isolated from mustached monkeys (SIVmus-1 and SIVmus-2) (Figure 1C). We also estimated the phylogenetic position of SIVrtg_Kib using SIV lineages with complete genomes. Briefly, nucleotide sequences of $g a g$, pol, env and nef were codon aligned individually using ClustalW and edited manually, followed by Bayesian analysis using the BEAST v1.6.2 program [22]. The Bayesian phylogeny shows that across all four genes examined, SIVrtg_Kib forms a separate lineage that clusters with the other Cercopithecus-specific SIVs and SIVtal (Figure 2). Within the Cercopithecus SIV group, SIVrtg_$\mathrm{Kib}$ is ancestral to the SIVgsn/mon/mus lineage in each gene with strong support, which includes SIVs from greater spot-nosed guenons (SIVgsn; $C$. nictitans), mona monkeys (SIVmon; C. mona) and mustached monkeys (SIVmus-1 and SIVmus-2).

In 2004, Verschoor et al. described the discovery of SIVasc_Qu from a red-tailed guenon in a zoo in the Netherlands [23]. This virus was isolated from the same subspecies in which we discovered SIVrtg_Kib in Kibale National Park, however the authors were only able to recover a 1895-bp partial pol sequence. In 2011, AhukaMundeke and colleagues discovered a second SIV from red-tailed guenons in the Democratic Republic of Congo (SIVasc_DRC), although from a different sub-species, Cercopithecus ascanius whitesidei [13]. Ahuka-Mundeke et al. were able to only recover a 648-bp partial pol fragment, probably due to limitations associated with nucleic acid recovery from bushmeat. Because full genomes were unavailable for both viruses, we did not include them in our sliding window similarity plot analysis. We did, however, determine the pairwise genetic distance of SIVrtg_Kib to SIVasc_Qu and other Cercopithecus SIVs by aligning corresponding sequences to the $1895 \mathrm{nt}$ partial pol sequence initially recovered from SIVasc_Qu. Our new SIVrtg_Kib is as divergent from SIVasc_Qu as from the other Cercopithecus SIVs, sharing $66 \%$ identity with SIVasc while sharing $65.6 \pm 1.8 \%$ identity with the remaining SIVs in this genus. The high degree of sequence divergence could be a result of the vast geographical range of $C$. ascanius schmidti, stretching from the Congo-Oubangui River system in central Africa, through Uganda to the Rift Valley in Kenya and western Tanzania [24]. Similarly, a pairwise comparison of SIVasc_DRC to SIVrtg_Kib revealed that both viruses were equally divergent, sharing $69.8 \%$ nucleotide identity. We also performed a separate phylogenetic

Table 3 Percent nucleotide identity of concatenated Gag-Pol-Env-Nef sequences along the coding region for SIVrtg_Kib and other major SIV lineages

\begin{tabular}{|c|c|c|c|c|c|c|c|c|c|c|c|c|c|}
\hline \multicolumn{14}{|c|}{ Nucleotide identity [\%] } \\
\hline SIV strains & rtg_Kib & mus-1 & mus-2 & deb & tal & $\mathrm{cpz}$ & syk & mnd-2 & agm & $\mathrm{rcm}$ & smm & Ist & col \\
\hline rtg_Kib & 100 & & & & & & & & & & & & \\
\hline mus-1 & 63.2 & 100 & & & & & & & & & & & \\
\hline mus-2 & 62.6 & 75.1 & 100 & & & & & & & & & & \\
\hline deb & 55.1 & 55.3 & 54.9 & 100 & & & & & & & & & \\
\hline tal & 57.5 & 56.6 & 56.1 & 57.8 & 100 & & & & & & & & \\
\hline cpz & 56.1 & 56.4 & 56.5 & 52.5 & 52.3 & 100 & & & & & & & \\
\hline syk & 54.4 & 53.1 & 54.3 & 59.8 & 56.1 & 51.1 & 100 & & & & & & \\
\hline mnd-2 & 52.3 & 52.0 & 52.3 & 54.0 & 54.6 & 54.3 & 53.0 & 100 & & & & & \\
\hline agm & 53.6 & 51.9 & 52.5 & 53.8 & 55.6 & 52.5 & 54.5 & 57.6 & 100 & & & & \\
\hline $\mathrm{rcm}$ & 52.7 & 51.7 & 51.7 & 53.8 & 54.8 & 54.5 & 54.0 & 63.9 & 59.0 & 100 & & & \\
\hline smm & 52.8 & 52.7 & 53.1 & 55.1 & 55.5 & 52.7 & 54.0 & 59.2 & 58.4 & 61.4 & 100 & & \\
\hline Ist & 50.8 & 50.0 & 50.1 & 52.1 & 52.4 & 50.3 & 51.9 & 57.0 & 55.1 & 55.5 & 55.1 & 100 & \\
\hline col & 47.8 & 48.1 & 47.4 & 48.5 & 47.6 & 47.9 & 47.0 & 49.4 & 49.3 & 48.9 & 48.8 & 48.8 & 100 \\
\hline
\end{tabular}




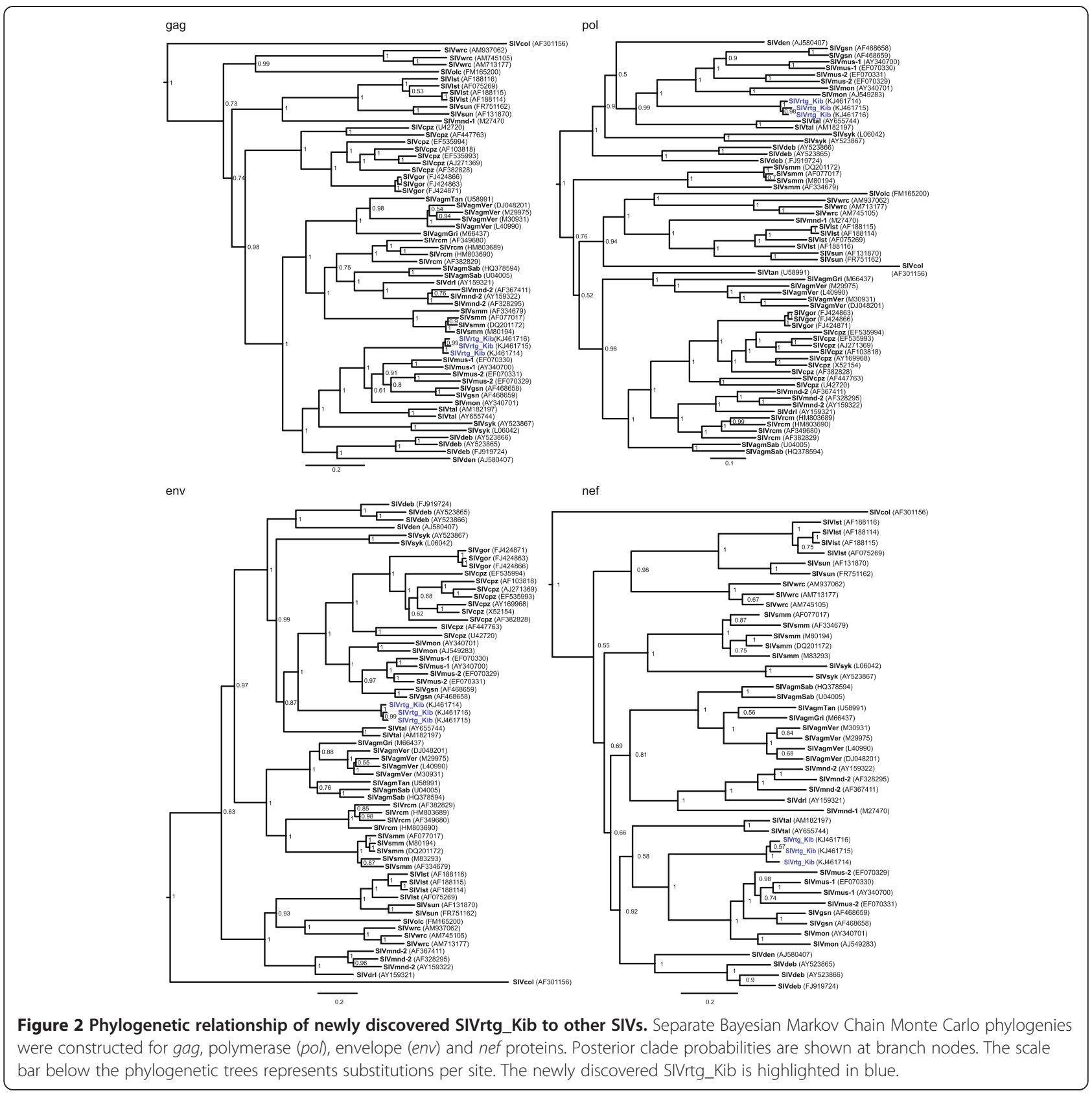

analysis that included all three SIVs isolated from redtailed guenons as well as other representative SIV lineages and determined the time to most recent common ancestor (TMRCA) using Bayesian inference and calibration of the molecular clock using an the estimated 10,000 year old separation of the drill (Mandrill leucophaeus) SIVs on mainland Africa from those on Bioko Island, Equatorial Guinea, as previously described [8]. The root of the tree is estimated to be 33,394 years before present (ybp) (95\% highest posterior density $(\mathrm{HPD})=19,157-51,174 \mathrm{ybp}$ ) and is thus comparable to that inferred for the Bioko monkey SIV phylogenies $(49,129$ ybp; $95 \% \mathrm{HPD}=29,078$ -
71,268 ybp) [25] (Figure 3). While SIVrtg_Kib groups together with SIVasc_Qu and SIVasc_DRC, there is a split between SIVrtg_Kib and SIVasc_Qu/DRC that occurred at least $15,500 \mathrm{ybp}(95 \% \mathrm{HPD}=7554-15,883 \mathrm{ybp})$. We also acknowledge that despite the use of a strong geological calibration point for our molecular dating estimates, considerable debate exists about the accuracy of SIV TMRCA estimates and suggest that dates should be regarded as minimum estimates. Evidence of genetic recombination in the SIVrtg-Kib was not observed in either bootscan analysis using the Simplot program (data not shown) or in the topologies inferred for each of the major gene regions and 


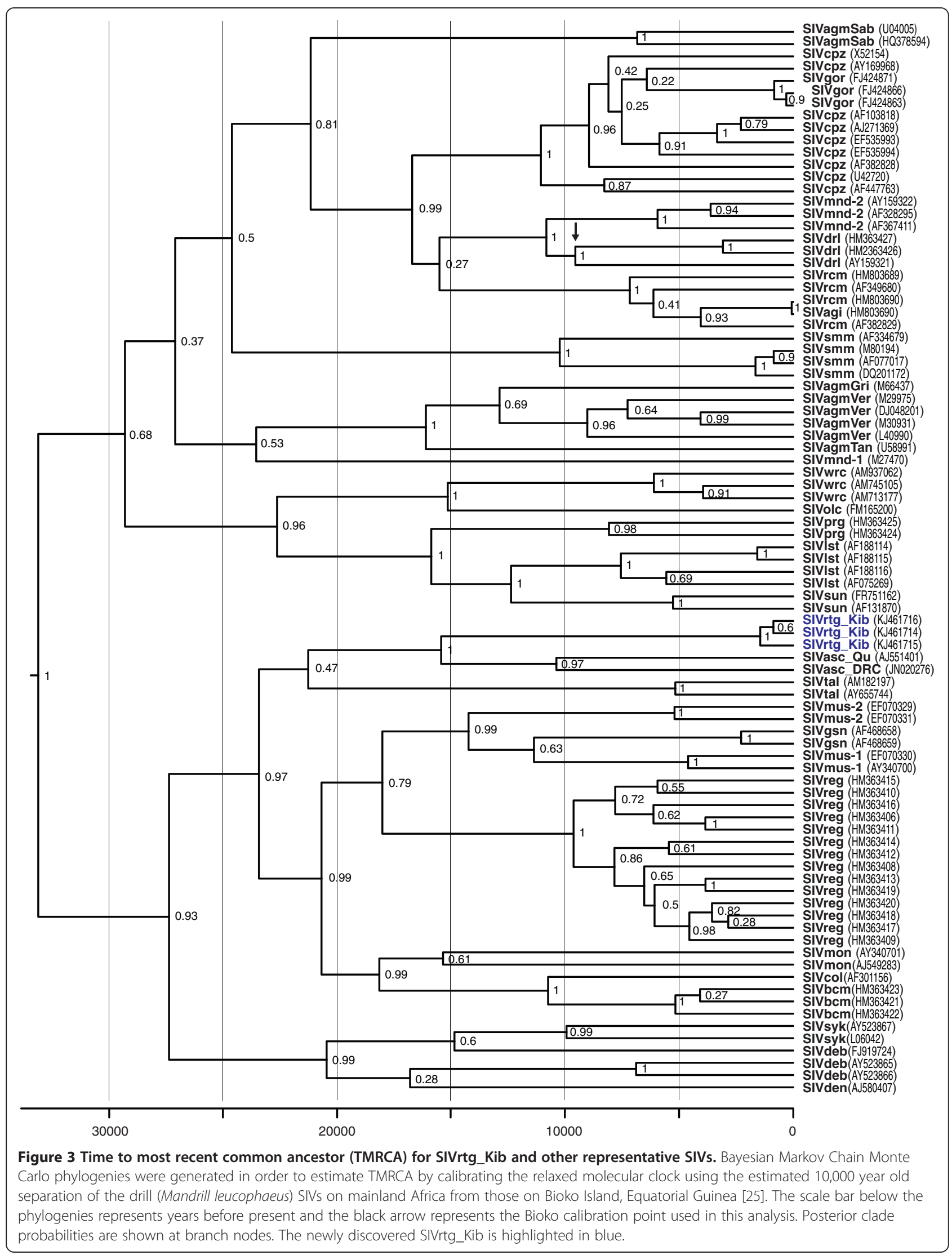


thus recombination likely did not influence the phylogenetic analyses.

In this study, we describe the first full-length SIV genome sequence isolated from the blood plasma of wildcaught red-tailed guenons. The high diversity observed among SIVs isolated from this species, as well as from other Cercopithecus SIVs from West and Central Africa, could potentially be explained by geographic separation of host species. Guenons are distributed throughout most of Sub-Saharan Africa and live in populations separated by large distances or geographic barriers [6]. As a consequence, limited contact between red-tailed guenons from different areas of Africa could limit the transmission of SIVs among guenon populations. Alternatively, crossspecies transmission among different guenon taxa may have contributed to SIV diversity, which would not be surprising given that sympatric guenons are known to hybridize in both wild and captive settings. The complex evolutionary relationships among taxa within the genus Cercopithecus suggest that additional sampling of the guenons may be fruitful for understanding the co-evolution of SIVs and their hosts.

\section{Competing interests}

The authors declare that they have no competing interests.

\section{Authors' contributions}

Conceived and designed the experiments: DHO TLG TCF NT CAC WMS ML. Performed the experiments: ML SDS WMS AS HZ. Analyzed the data: ML WMS JMG AJE DHO. Wrote the paper: ML WMS TLG DHO TCF. Conducted study in the field: DH AT GW TLG. All authors read and approved the final manuscript.

\section{Acknowledgements \\ This work was funded by NIH grant TW009237 as part of the joint NIH-NSF Ecology of Infectious Disease program and the UK Economic and Social Research Council and in part by National Institutes of Health grants R01 Al084787 and R01 Al077376-04A1; it was also supported by National Center for Research Resources grant RR000167 and the Office of Research Infrastructure Programs (ORIP) grant P510D011106. The research was conducted, in part, at a facility constructed with support from Research Facilities Improvement Program grants RR15459-01 and RR020141-01. Use of trade names is for identification only and does not imply endorsement by the U.S. Department of Health and Human Services, the Public Health Service, or the Centers for Disease Control and Prevention. The findings and conclusions in this report are those of the authors and do not necessarily represent the views of the Centers for Disease Control and Prevention.}

\footnotetext{
Author details

${ }^{1}$ Wisconsin National Primate Research Center, 555 Science Dr, 53705 Madison, WI, USA. 'Laboratory Branch, Division of HIV/AIDS Prevention, National Center for HIV, Hepatitis, STD, and TB Prevention, Centers for Disease Control and Prevention, Atlanta, GA, USA. ${ }^{3}$ Department of Pathobiological Sciences, University of Wisconsin-Madison, Madison, WI, USA. ${ }^{4}$ Makerere University, Kampala, Uganda. ${ }^{5}$ Department of Anthropology and Institute of Ecology and Evolution, University of Oregon, Eugene, OR, USA. ${ }^{6}$ Department of Anthropology and School of Environment, McGill University, Montreal, QC, Canada. Wildlife Conservation Society, Bronx, New York, USA. ${ }^{8}$ Department of Pathology and Laboratory Medicine, University of Wisconsin-Madison, Madison, WI, USA.
}

Received: 18 February 2014 Accepted: 24 June 2014

Published: 4 July 2014

\section{References}

1. Locatelli S, Peeters M: Cross-species transmission of simian retroviruses: how and why they could lead to the emergence of new diseases in the human population. AIDS 2012, 26:659-673.

2. Gao F, Bailes E, Robertson DL, Chen Y, Rodenburg CM, Michael SF, Cummins LB, Arthur LO, Peeters M, Shaw GM, Sharp PM, Hahn BH: Origin of HIV-1 in the chimpanzee Pan troglodytes troglodytes. Nature 1999, 397:436-441.

3. Hirsch VM, Olmsted RA, Murphey-Corb M, Purcell RH, Johnson PR: An African primate lentivirus (SIVsm) closely related to HIV-2. Nature 1989, 339:389-392.

4. Bibollet-Ruche F, Bailes E, Gao F, Pourrut X, Barlow KL, Clewley JP, Mwenda JM, Langat DK, Chege GK, McClure HM, Mpoudi-Ngole E, Delaporte E, Peeters M, Shaw GM, Sharp PM, Hahn BH: New simian immunodeficiency virus infecting De Brazza's monkeys (Cercopithecus neglectus): evidence for a cercopithecus monkey virus clade. J Virol 2004, 78:7748-7762.

5. Liegeois F, Courgnaud V, Switzer WM, Murphy HW, Loul S, Aghokeng A, Pourrut X, Mpoudi-Ngole E, Delaporte E, Peeters M: Molecular characterization of a novel simian immunodeficiency virus lineage (SIVtal) from northern talapoins (Miopithecus ogouensis). Virology 2006, 349:55-65.

6. Guschanski K, Krause J, Sawyer S, Valente LM, Bailey S, Finstermeier K, Sabin R, Gilissen E, Sonet G, Nagy ZT, Lenglet G, Mayer F, Savolainen V: Next-generation museomics disentangles one of the largest primate radiations. Syst Biol 2013, 62:539-554.

7. Goldberg TL, Sintasath DM, Chapman CA, Cameron KM, Karesh WB, Tang S, Wolfe ND, Rwego IB, Ting N, Switzer WM: Coinfection of Ugandan red colobus (Procolobus [Piliocolobus] rufomitratus tephrosceles) with novel, divergent delta-, lenti-, and spumaretroviruses. J Virol 2009, 83:11318-11329.

8. Lauck M, Switzer WM, Sibley SD, Hyeroba D, Tumukunde A, Weny G, Taylor B, Shankar A, Ting N, Chapman CA, Friedrich TC, Goldberg TL, O'Connor DH: Discovery and full genome characterization of two highly divergent simian immunodeficiency viruses infecting black-and-white colobus monkeys (Colobus guereza) in Kibale National Park. Uganda Retrovirol 2013, 10:107.

9. Goldberg TL, Paige SB, Chapman CA: The Kibale EcoHealth Project: exploring connections among human health, animal health, and landscape dynamics in western Uganda. In New Directions in Conservation Medicine: Applied Cases of Ecological Health. Edited by Aguirre PD AA, Ostfeld RS. New York: Oxford University Press; 2012:452-465.

10. Lauck M, Hyeroba D, Tumukunde A, Weny G, Lank SM, Chapman CA, O'Connor DH, Friedrich TC, Goldberg TL: Novel, divergent simian hemorrhagic fever viruses in a wild Ugandan red colobus monkey discovered using direct pyrosequencing. PLoS One 2011, 6:e19056.

11. Lauck M, Sibley SD, Hyeroba D, Tumukunde A, Weny G, Chapman CA, Ting $\mathrm{N}$, Switzer WM, Kuhn JH, Friedrich TC, O'Connor DH, Goldberg TL: Exceptional simian hemorrhagic fever virus diversity in a wild african primate community. J Virol 2013, 87:688-691.

12. Aghokeng AF, Bailes E, Loul S, Courgnaud V, Mpoudi-Ngolle E, Sharp PM, Delaporte E, Peeters M: Full-length sequence analysis of SIVmus in wild populations of mustached monkeys (Cercopithecus cephus) from Cameroon provides evidence for two co-circulating SIVmus lineages. Virology 2007, 360:407-418

13. Ahuka-Mundeke $S$, Ayouba A, Mbala-Kingebeni P, Liegeois F, Esteban A, Lunguya-Metila O, Demba D, Bilulu G, Mbenzo-Abokome V, Inogwabini Bl, Muyembe-Tamfum JJ, Delaporte E, Peeters M: Novel multiplexed HIV/simian immunodeficiency virus antibody detection assay. Emerg Infect Dis 2011, 17:2277-2286.

14. Aghokeng AF, Ayouba A, Mpoudi-Ngole E, Loul S, Liegeois F, Delaporte E, Peeters M: Extensive survey on the prevalence and genetic diversity of SIVs in primate bushmeat provides insights into risks for potential new cross-species transmissions. Infection, Genetics and Evolution 2010, 10:386-396.

15. Courgnaud V, Salemi M, Pourrut X, Mpoudi-Ngole E, Abela B, Auzel P, Bibollet-Ruche F, Hahn B, Vandamme AM, Delaporte E, Peeters M: Characterization of a novel simian immunodeficiency virus with a vpu gene from greater spot-nosed monkeys (Cercopithecus nictitans) provides new insights into simian/human immunodeficiency virus phylogeny. J Virol 2002, 76:8298-8309.

16. Dazza MC, Ekwalanga M, Nende M, Shamamba KB, Bitshi P, Paraskevis D, Saragosti S: Characterization of a novel vpu-harboring simian immunodeficiency virus from a Dent's Mona monkey (Cercopithecus mona denti). J Virol 2005, 79:8560-8571. 
17. Emau P, McClure HM, Isahakia M, Else JG, Fultz PN: Isolation from African Sykes' monkeys (Cercopithecus mitis) of a lentivirus related to human and simian immunodeficiency viruses. J Virol 1991, 65:2135-2140.

18. Altschul SF, Gish W, Miller W, Myers EW, Lipman DJ: Basic local alignment search tool. J Mol Biol 1990, 215:403-410.

19. Locatelli S, Lafay B, Liegeois F, Ting N, Delaporte E, Peeters M: Full molecular characterization of a simian immunodeficiency virus, SIVwrcpbt from Temminck's red colobus (Piliocolobus badius temminckii) from Abuko Nature Reserve, The Gambia. Virology 2008, 376:90-100

20. Lole KS, Bollinger RC, Paranjape RS, Gadkari D, Kulkarni SS, Novak NG, Ingersoll R, Sheppard HW, Ray SC: Full-length human immunodeficiency virus type 1 genomes from subtype C-infected seroconverters in India, with evidence of intersubtype recombination. J Virol 1999, 73:152-160.

21. Abascal F, Zardoya R, Telford MJ: TranslatorX: multiple alignment of nucleotide sequences guided by amino acid translations. Nucleic Acids Res 2010, 38:W7-13.

22. Drummond AJ, Rambaut A: BEAST: Bayesian evolutionary analysis by sampling trees. BMC Evol Biol 2007, 7:214.

23. Verschoor EJ, Fagrouch Z, Bontjer I, Niphuis H, Heeney JL: A novel simian immunodeficiency virus isolated from a Schmidt's guenon

(Cercopithecus ascanius schmidti). J Gen Virol 2004, 85:21-24.

24. Oates JF, Hart J, Groves CP, Butynski TM: The International Union for the Conservation of Nature (IUCN) red list. 2008. 3.1.

25. Worobey M, Telfer P, Souquiere S, Hunter M, Coleman CA, Metzger MJ, Reed P, Makuwa M, Hearn G, Honarvar S, Roques P, Apetrei C, Kazanji M, Marx PA: Island biogeography reveals the deep history of SIV. Science 2010, 329:1487.

doi:10.1186/1742-4690-11-55

Cite this article as: Lauck et al:: Discovery and full genome characterization of a new SIV lineage infecting red-tailed guenons (Cercopithecus ascanius schmidti) in Kibale National Park, Uganda. Retrovirology 2014 11:55.

\section{Submit your next manuscript to BioMed Central and take full advantage of:}

- Convenient online submission

- Thorough peer review

- No space constraints or color figure charges

- Immediate publication on acceptance

- Inclusion in PubMed, CAS, Scopus and Google Scholar

- Research which is freely available for redistribution 